\title{
DESERCIÓN EN LA EDUCACIÓN SUPERIOR RECINTO LAS MINAS. PERÍODO 2001-2007
}

Leonor Ruiz Calderón ${ }^{[5]}$

\section{Resumen}

El estudio sobre la deserción en la Educación Superior se realizó en la Universidad de las Regiones Autónomas de la Costa Caribe Nicaragüense -URACCAN - Recinto Las Minas, comprende tres extensiones: Rosita, Bonanza y Waslala, e incluye la sede central en Siuna. Está enfocado a dimensionar la magnitud del problema de la deserción y la construcción de propuestas tanto a nivel institucional, personal y social.

Las interrogantes que guiaron la presente investigación fueron: ¿Cuáles son los índices de deserción y tasa de titulación de URACCAN Las Minas?, ¿Qué factores personales y/o familiares están asociados a la deserción? y ¿Cuáles son las implicancias que tiene en los desertores y el costo para la universidad?

Se ofrece un panorama descriptivo en el paradigma cuantitativo de la deserción para responder al planteamiento del problema. La dimensión es de corte transversal, retrospectivo y propositivo porque contiene un plan de seguimiento al problema de la deserción. Para fines del estudio se utilizó datos estadísticos de la oficina de registro estudiantil. Se diseñó un cuestionario para ser aplicado a las personas desertoras a una muestra del $25 \%$ del universo que corresponde a 662, técnica de elección al azar, de una lista de desertores correspondientes a los años objeto de estudio.

Los datos encontrados en relación al índice de deserción fue del 15.95\%. Los factores que influyen son el económico, laboral y académico. Las implicancias identificadas son sociales e institucionales. Los datos evidencian el índice de deserción, los factores que inciden, las implicaciones tanto para la universidad y el país en general.

Palabras Claves: deserción, pregrado, implicancias, educación.

[5] Máster en Antropología. Docente investigadora de la URACCAN, Recinto Las Minas.

30 | CIenCia e interCulturalidad, Volumen 4, Año 2, No. 2, Junio 2009 


\section{Introducción}

La deserción escolar es un problema que se manifiesta, en los tres niveles del sistema educativo de todos los países de América Latina y el Caribe. Muchos universitarios en Nicaragua abandonan sus estudios por limitaciones económicas, aunque otros lo hacen porque no pueden cumplir las exigencias académicas; el mismo oscila entre un 30 y 40 por ciento, cifras que para algunos nicaragüenses no es alarmante. Miles de universitarios tienen que abandonar la universidad todos los años, constituye un problema que afecta enormemente a las Universidades (IESALC-UNESCO). Citado por Sandino, (2006: 10).

De una población estudiantil aproximada de 189,832 en 52 instituciones de Educación Superior, un 60 por ciento son atendidos por las universidades privadas que no son miembros del Consejo Nacional de Universidades -CNU-, y cuyo crecimiento se ha duplicado en los últimos seis años, pasa de 21 en el 2001 a 43 en el 2006. Evidentemente, la mayor participación de la demanda estudiantil está en las universidades privadas que se encuentran representadas por el Consejo Superior de Universidades Privadas (COSUP) y la Federación Nacional de Universidades Privadas (FENUP), instituciones que aún no hacen públicos sus indicadores oficiales (Ibíd.).

URACCAN se constituyó en 1992 y fue legalizada en 1993 a través de la autorización del Consejo Nacional de Universidades y la Asamblea Nacional. Para la organización se estructura en Comités de Apoyo en los Municipios de ambas Regiones Autónomas, donde se designan a personas para conformar una Asociación que trabajó los aspectos políticos y organizativos de la Universidad.

En el seno de esa instancia se acordó establecer la Universidad en sedes ubicadas en ambas Regiones en aras de mantener la unidad multiétnica costeña. URACCAN inicia operaciones en enero de 1995 con el establecimiento del Programa de Educación a Distancia (PRUEDIS) para 120 docentes de ambas Regiones Autónomas de Nicaragua, en coordinación con la Universidad Nacional Autónoma de Nicaragua - UNANManagua-. Del $75 \%$ del total de los estudiantes matriculados, $45 \%$ fueron mujeres y 9.77\% indígenas. La procedencia étnica de los estudiantes fue: Miskito 3\%, Mayangna $6 \%$, Rama $0.77 \%$, Creoles $13 \%$, mestizos $77 \%$, garífunas $0.68 \%$.

La URACCAN es la única Universidad del país que cuenta con cifras de matriculas según grupos étnicos, con la tasa de estudiantes indígenas que ha aumentado de 58 a 751 entre 1995 y 2005. La matrícula total para el 2004 fue aproximadamente de 4.400 estudiantes (Ibíd. pág. 2). 


\section{LA EDUCACIÓN SUPERIOR EN LA REGIÓN AUTÓNOMA ATLÁNTICO NORTE}

La investigación desarrollada por Salgado, R. y Cruz, C. (2004:28), sobre los factores que influyeron en la deserción escolar, Recinto Siuna, correspondiente al período 1995-2000 refleja, que de un total de 1059 estudiante ingresados, se retiraron 178, lo que representa un $17 \%$ como promedio anual.

En el mismo trabajo, se concluye que las carreras con más estudiantes que abandonaron los estudios están representadas por Ciencias de la Educación e Ingeniería Agroforestal, en su totalidad el mayor abandono lo representan las mujeres con el $76 \%$. Como factores relevantes del fenómeno plantean: el bajo rendimiento estudiantil, y las condiciones mínimas de la URACCAN en los primeros años de funcionamiento.

A pesar de conocer la problemática del abandono escolar que se experimenta en cada año, no existe un estudio que indique las causas fundamentales de esta grave situación, por ello es la necesidad de indagar en este tema para lo cual se plantean las interrogantes: ¿Cuáles son los índices de deserción y tasa de titulación de URACCAN Las Minas?, ¿Qué factores están asociados a la deserción? ¿Cuáles son las implicancias que tiene en los desertores y el costo para la universidad?

\section{Revisión de literatura}

\section{Î́ndices de la deserción y tasa de titulación}

\section{Cálculo de la deserción}

En el documento preparado para reunión de rectores de universidades Panameñas se presentaron estudios de repitencia y deserción en América Latina. Explica el documento que se tomó para cada carrera la cohorte que ingresó tres años antes que el período normal de duración de la carrera. Por ejemplo, si la carrera es de 4 a 5 años de duración se toma la cohorte que ingresó hace siete años. Luego se establece para dichas cohortes la cantidad de estudiantes que se titularon en el año (t), que correspondía al período normal de duración de la carrera (d), la cantidad que se titularon un año más tarde $(t+1)$, dos años más tarde $(t+2)$ y tres años después $(t+3)$ respectivamente, $y$ aquellos que aún no se titulan y siguen estudiando. Los estudiantes de la cohorte de ingreso que no estaban en ninguna de las categorías anteriores, corresponden a los desertores (IESALC/UNESCO, 2006:11). 


\section{Eficiencia de Titulación}

La eficiencia de titulación "E" del sistema, se define como la proporción de estudiantes "T" que se titulaba en un año " $t$ ", en comparación con la matrícula nueva en primer año "N", en el tiempo correspondiente a una duración "d" de las carreras establecidas en los planes de estudios oficiales. Es decir: $\mathrm{E}=\mathrm{T}(\mathrm{t}) / \mathrm{N}(\mathrm{t}-\mathrm{d}$ ). (Ibíd.).

Para calcular la duración promedio de las carreras, en caso de que no se pudiera obtener el dato exacto, se hace una estimación de las carreras de pregrado (licenciaturas), en cinco años.

Estudios realizados por la Universidad Católica de Chile demuestran que la deserción académica presenta un crecimiento mayor de $17.7 \%$ que la deserción voluntaria (8.3\%) a lo largo de ocho semestres considerados (Méndez, 1998:29).

De acuerdo a cifras de la dirección de planeación de Costa Rica en los últimos ciclos escolares se ha reflejado una sensible mejoría en los niveles de reprobación y deserción escolar que alcanzaron un $15.3 \%$ y $18 \%$ respectivamente en el ciclo escolar (2000-2001) y en el (2001-2002), ambos índices se ubicaron en un $43.7 \%$ y un $17.5 \%$ respectivamente lo que significa que el porcentaje de reprobación disminuye con el $1.6 \%$ y la deserción escolar el $0.6 \%$; indicadores que reflejan una mejoría en la calidad de la educación (Ibíd.: 12).

Aun con la gran cantidad de matrícula en las universidades, un 50\% de la deserción estudiantil se produce en los primeros años de estudios superior por esta razón se ha creado un ciclo común que permita mejorar la calidad de Educación Superior fundamentalmente en estos primeros años donde más deserción o fracasos existen en el ciclo superior (Jaramillo, 1999:15).

Es importante destacar que el mayor retorno por año adicional que obtienen las mujeres en comparación con los hombres en los mercados laborales explica que los costos privados de la deserción sean, en los términos señalados más altos para ellas (44\% y $23 \%$ ) respectivamente. Las diferencias constatadas entre mujeres y hombres en materia de menores ingresos obtenidos al abandonar con antelación la escuela, indican que la elevación de las tazas de retención escolar en el caso de estas genera mayores incrementos de ingresos a lo largo de su vida activa en comparación con los varones (García, op.cit, p. 16).

\section{Clasificación de la Deserción}

La deserción se clasifica en dos momentos, de acuerdo al tiempo y el espacio. 


\section{LA EDUCACIÓN SUPERIOR EN LA REGIÓN AUTÓNOMA ATLÁNTICO NORTE}

De acuerdo al tiempo puede ser:

Deserción precoz: en donde la persona que habiendo sido aceptada por la universidad no se matricula.

Deserción temprana: abandona sus estudios en los cuatro primeros semestres de la carrera y la deserción tardía, abandona los estudios en los últimos seis semestres, es decir, a partir del quinto semestre en adelante (Ibíd.: 17).

De acuerdo al espacio:

Deserción institucional: es el caso, en el cual el estudiante abandona la universidad.

Deserción interna o del programa académico: Cuando el estudiante decide cambiar su programa académico por otro que ofrece la misma institución universitaria (Ibíd.).

\section{Factores asociados al abandono en la educación superior}

Brea, (2004: 5) destaca, sobre las condiciones laborales y por ende las horas de dedicación al estudio - son estudiantes que disponen de poco de tiempo para el estudio-; así también, los problemas motivacionales, personales y psico-afectivos que provocaron el fracaso académico, tales como, los sentimientos de frustración, desorientación vocacional, baja autoestima y la posible y precaria adaptación al medio universitario.

\section{a) Factores exógenos}

Está relacionado al lugar de residencia, el nivel socioeconómico, nivel educativo de los padres, la condición de actividad económica del estudiante.

La formación académica previa, el nivel de aprendizaje adquirido, éxito, fracaso, deficiencia en la educación media, aspiraciones y motivaciones personales, las propias aptitudes, grado de satisfacción con la carrera, relación de la carrera con el mercado laboral, dificultades personales para la integración y la adaptación, las experiencias del estudiante en el aula, el apoyo y la orientación recibido por los profesores, el nivel educativo de los padres: Existe una asociación significativa entre la intencionalidad de continuar estudios de nivel superior y el nivel educativo de los padres. El $86 \%$ de quienes tienen padres con estudios universitarios pretende seguir en una universidad. 
Distancia entre la formación previa de los integrantes y las competencias y conocimientos que se requieren para los estudios universitarios: baja calidad de los aprendizajes adquiridos, dificultades para la comprensión de textos y la escritura, dificultades para abordar abstracciones simples y/o complejas, dificultades para resolver problemas elementales, escaso capital cultural, conocimientos insuficientes en disciplinas como matemática, química, biología y física.

\section{b) Factores endógenos}

Estos factores están asociados a la existencia de políticas de admisión, (Irrestricto o directo, selectivo sin cupo, selectivo con cupo), presencia de políticas de orientación vocacional, excesivo número de programas en oferta, tipo de carrera y relación con la formación previa del estudiante, la duración de las carreras (duración teórica inferior a la educación real que se incrementa dos o tres años), el currículo, dedicación del alumno (no siempre es full time), calidad/ pertinencia pedagógica del cuerpo docente y el equipamiento.

Escobar, Delgado, Fruto, y Hernández, (2005: 4) exteriorizan en su estudio sobre la deserción y repitencia en la Educación Superior en Panamá; tres factores como causas fundamentales de la discontinuidad de los estudio en el medio universitario estudiantes:

a) Factores Socioeconómicos: Pobre vocación hacia la carrera escogida, las y los estudiantes que provienen de hogares en condiciones socios económicos desfavorables tienden a desertar más y la necesidad financiera y la decisión entre trabajar y estudiar, resaltan como causas.

b) Factores Institucionales: Insatisfacción con la calidad y asistencia de los profesores, con las facilidades de la universidad, evaluación injusta o inadecuada, limitación para satisfacer aspectos de seguridad, soporte y apoyo de sus miembros y la falta de planificación y consistencia en la política educativa..

c) Factores Académicos: Se consideran de interés la situación de las bajas calificaciones, pérdida de interés por los estudios, deficiente formación de la Educación Media, lo que se refleja en una alta tasa de reprobación en primer año.

A mayor promedio de secundaria, mayor rendimiento académico universitario.

Mayor rendimiento de los estudiantes que provienen de los colegios privados, que los estudiantes egresados de colegios públicos. 


\section{LA EDUCACIÓN SUPERIOR EN LA REGIÓN AUTÓNOMA ATLÁNTICO NORTE}

\section{Implicancias de la deserción}

En cuanto a las implicancias se pueden distinguir tres categorías: las sociales, institucionales y personales.

\section{Personales: Disonancia con aspiraciones, trayectoria laboral}

Entre las personales está el disgusto, la frustración y la sensación de fracaso de los repitentes y desertores con los consiguientes efectos en su salud física y mental. Asimismo, se produce una pérdida de oportunidades laborales dadas las menores posibilidades de conseguir empleos satisfactorios, la postergación económica por salarios más bajos, con los consiguientes impactos en los costos en términos individuales y familiares (IESALC/UNESCO, Ibíd.: 19).

\section{Institucionales: Costos y prestigio}

Una de las implicancias más importantes de la repitencia y la deserción es el costo que ello implica para los países. La debilidad de los datos hace muy difícil el cálculo de dichos costos. En un estudio reciente de UNESCO en 15 países que cubren un 90\% de la Región el costo de la deserción se estimaba en U\$11,1 billones de dólares al año, lo que en países como Brasil equivale al costo de 2 millones de estudiantes universitarios (Ibíd.).

Para calcular el promedio anual de los costos directos por estudiante, hay dos situaciones diferentes. Para las instituciones estatales se asume que un $75 \%$ de los fondos que aporta el Estado a las universidades se destina a docencia, lo cual, al conocerse la matrícula total, permite estimar un costo por estudiante. Para las universidades privadas se puede asumir que el costo anual promedio por estudiante "CPAP" es igual a lo que los alumnos cancelan anualmente (Ibíd.).

También está la limitación para cumplir la misión institucional y un descenso en los índices de eficiencia y calidad. De igual manera se tiene implicancias económicas debido a los menores ingresos por matrícula y a los costos adicionales para las universidades tanto públicas como privadas. Implica además, una disminución del rendimiento académico de la universidad y un incremento innecesario del número de estudiantes (Ibíd.). 


\section{Sociales: gasto en educación y equidad}

Entre las sociales está la retroalimentación del círculo de la pobreza y la gestación de una "capa social" de frustrados profesionales con posible disminución del aporte intelectual y el potencial aumento del subempleo. Adicionalmente se incrementa el costo para el país de la educación, asociado a una sub - optimización de los recursos debido al costo de la deserción (Ibíd.: 20).

También contribuye a generar inequidad y desequilibrios sociales y desvirtúa los objetivos que la sociedad le ha entregado a la Educación Superior (Ibíd.).

\section{Materiales y métodos}

Para fines del estudio se utilizaron datos estadísticos de la oficina de registro estudiantil. Se analizaron cifras estadísticas de los años 2001 al 2007, en las diferentes carreras específicas de la Universidad en: Ingeniería Agroforestal, Zootecnia; Licenciaturas en Ciencias Sociales con mención en Desarrollo Local, Administración de Empresas con mención en Banca y Finanzas, Ciencias de la Educación con menciones en Historia, Biología, Español y Pedagogía.

Los datos son segregados por sexo, calculándose el índice de deserción en cada una de ellas. Se aplicó un cuestionario diseñado para desertores/as, a una muestra del $25 \%$ del universo que corresponde a 662, siendo personas elegidas al azar, de una lista de desertores correspondientes a los años 2001 al 2007. La encuesta recoge información sobre las condiciones generales, socioeconómicas y familiares de los estudiantes; los factores relacionados con el abandono de los estudios universitarios (económico, personal, familiar, motivacional, vocacional y académico); las implicaciones personales, sociales e institucionales que acarrea la deserción universitaria.

Este estudio es descriptivo bajo el paradigma cuantitativo, en la que se analizaron y vincularon datos en el mismo para responder al planteamiento del problema. La dimensión es de corte transversal, retrospectivo y propositivo que contiene un plan de seguimiento al problema de la deserción. Las variables que se utilizaron fueron el índice de deserción, eficiencia de titulación, factores que influyen en la deserción y sus implicancias.

El análisis de la información se efectuó de acuerdo con los objetivos planteados, valiéndose del fundamento teórico y los resultados encontrados en el estudio, así también valorando la experiencia y conocimientos del objeto de estudio de la autora de la investigación. 


\section{LA EDUCACIÓN SUPERIOR EN LA REGIÓN AUTÓNOMA ATLÁNTICO NORTE}

\section{Resultados y discusión}

En el gráfico 01 que se presenta a continuación, se puede apreciar que en el año 2001 con relación al 2002 se registró un descenso de $3.43 \%$, incrementándose en 5.1 al año siguiente. A partir del 2004 se mantuvo un descenso de 1.05 y finalmente en el 2007, drásticamente se registró un descenso del 6.85\%.

\section{ÍNDICE DE DESERCIÓN}

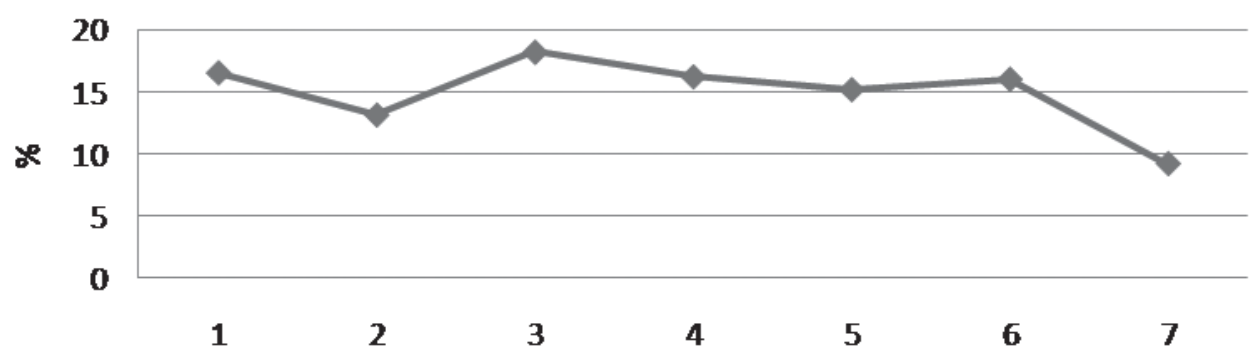

Gráfico 01: Índice de deserción Recinto Universitario Las Minas

En la carrera de Ingeniería en Zootecnia, es donde se registra el mayor porcentaje de deserción que es del 24.13\%, seguidamente Ciencias Sociales con mención en Desarrollo Local con un 16.1, Administración de Empresas 14.84, Ciencias de la Educación con mención en Historia 14.1; Ingeniería Agroforestal con 12.17\%, Pedagogía 11.26, Ciencias de la Educación con mención en Español, Educación Intercultural Bilingüe y Ciencias de la Educación con mención en Biología que presenta el más bajo con 4.17\%.

De acuerdo a la clasificación de la deserción, prevalece la deserción temprana, que corresponde a los estudiantes que abandonan los estudios en el primer semestre del primer año. De acuerdo al espacio es una deserción institucional, porque lo han hecho de la Educación Superior, no continúan en ninguna otra universidad. 


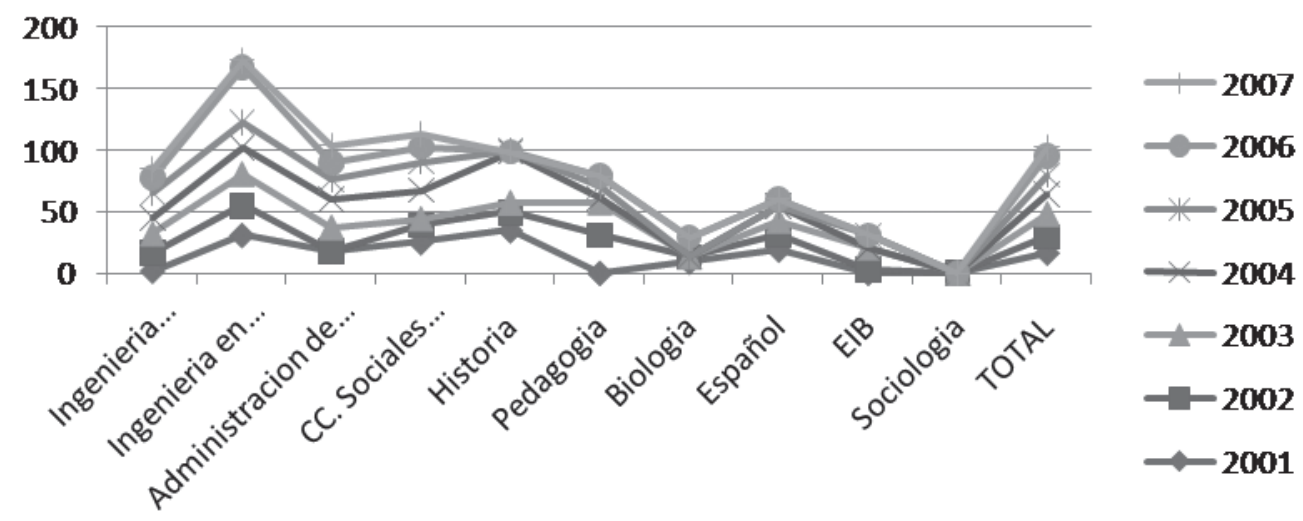

Figura 02: Deserción por año académico y por carrera ofertada

En el cuadro 01 que se muestra a continuación, se detalla la cantidad de estudiantes que atendió la Universidad con relación a la cantidad que finalizó. Desertaron durante el período, una cantidad de 662 estudiantes, destacándose las mujeres como las que más abandonan los estudios.

Cuadro 01: Tasa de deserción por sexo

\begin{tabular}{llllllll}
\hline \multirow{2}{*}{ PERÍODO } & \multirow{2}{*}{ Matrícula inicial } & Matrícula final & \multicolumn{3}{c}{ Deserción } & \multirow{2}{*}{$\%$} \\
\cline { 1 - 6 } $2001-2007$ & Total & Total & Total & F & M & \\
\cline { 2 - 6 } & 4.148 & 3.486 & 662 & 333 & 329 & 15.95 \\
\hline
\end{tabular}

Con relación a estudiantes egresados \as, se tiene una cantidad de 464, correspondiendo 260 a mujeres y 204 hombres. De este total, 335 lograron titularse en las diferentes carreras, corresponden 185 mujeres y 150 hombres. Hay curiosas diferencias por sexo y niveles de éxito. Se ha dicho que menos mujeres concluyen estudios superiores; no obstante, son más eficientes en materia de titulación que los hombres Se destaca que si bien es cierto que las mujeres son las que más desertan, también son las que más logran titularse. 


\section{LA EDUCACIÓN SUPERIOR EN LA REGIÓN AUTÓNOMA ATLÁNTICO NORTE}

Podría haber varias interpretaciones, una es que las mujeres que logran su ingreso, muestran mayor eficacia en el desarrollo de sus estudios. En cambio, generalmente por razones económicas y/o laborales, resultado de la necesidad de apoyar el ingreso familiar, los hombres suelen concluir sus estudios, pero sin obtener su título.

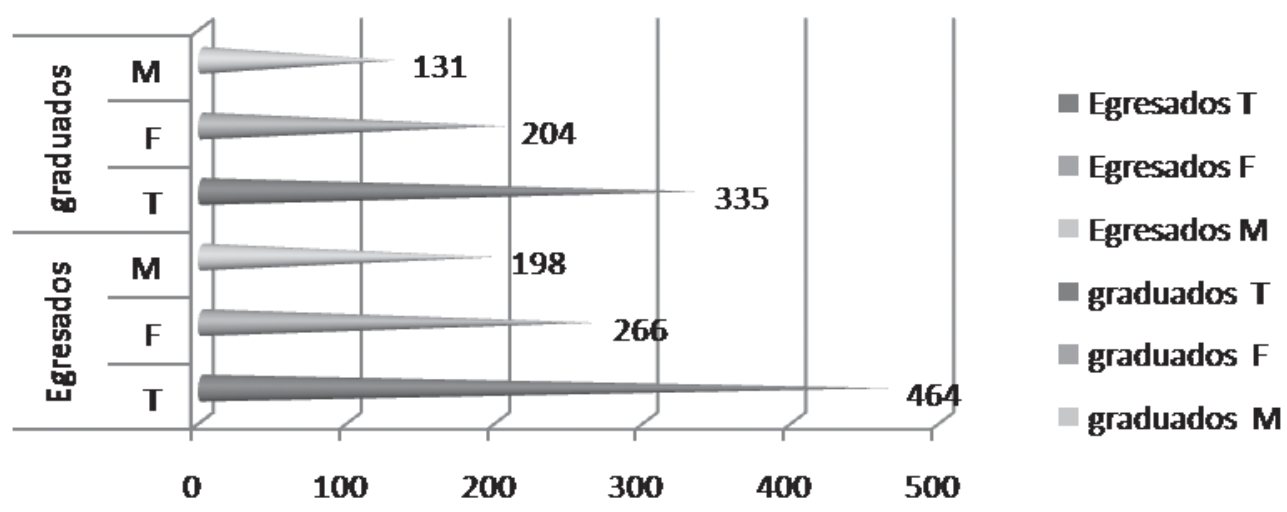

Figura 03: Egresados vis. Graduados

El comportamiento que ha tenido cada carrera con relación al total de egresados $\backslash$ as y titulados \as de acuerdo al período de duración de la carrera se puede apreciar en el cuadro que se presenta a continuación. En el caso de las Ingenierías, Administración de Empresas, Sociología y Ciencias de la Educación tienen una duración de cinco años. Ciencias Sociales con mención en Desarrollo Local es de cuatro años.

Se destaca la presencia de mayor número de mujeres en las carreras de Administración de Empresas, Ciencias Sociales y Educativas. En cambio, en las Ingenierías es donde más hombres han logrado titularse, donde la mayoría de estas carreras son más del sexo masculino.

El cuadro contiene información tomada desde el año 1999, período que la URACCAN tuvo los \as primeros egresados/as. 
Cuadro 2: Egresados y Titulados

\begin{tabular}{lllllll}
\hline \multirow{2}{*}{ CARRERAS } & \multicolumn{3}{c}{ Egresados } & \multicolumn{4}{c}{ graduados } \\
\cline { 2 - 8 } & \multicolumn{1}{c}{$\mathbf{T}$} & $\mathbf{F}$ & $\mathbf{M}$ & $\mathbf{T}$ & $\mathbf{F}$ & $\mathbf{M}$ \\
\hline Agroforestal & 102 & 31 & 71 & 81 & 19 & 62 \\
Zootecnia & 7 & 1 & 6 & 4 & 0 & 4 \\
Administración & 67 & 50 & 17 & 53 & 41 & 12 \\
CSS en Desarrollo Local & 95 & 61 & 34 & 47 & 29 & 18 \\
Sociología & 38 & 33 & 5 & 35 & 32 & 3 \\
Educación Intercultural & 33 & 8 & 25 & 17 & 6 & 11 \\
Ciencias de la Educación mención en Historia & 53 & 34 & 19 & 35 & 20 & 15 \\
Ciencias de la Educación mención en Biología & 36 & 25 & 11 & 31 & 22 & 9 \\
Pedagogía & 33 & 17 & 16 & 32 & 16 & 16 \\
\hline \multicolumn{1}{c}{ TOTAL } & $\mathbf{4 6 4}$ & $\mathbf{2 6 0}$ & $\mathbf{2 0 4}$ & $\mathbf{3 3 5}$ & $\mathbf{1 8 5}$ & $\mathbf{1 5 0}$ \\
\hline
\end{tabular}

Con relación al período objeto de estudio, un $72.20 \%$ de los egresados alcanzaron graduarse. Es notable mencionar que en el 2007 se registró un porciento mayor en relación a los años anteriores, que fue del $18.11 \%$ correspondiendo en su mayoría a nuevos/as profesionales en las carreras de Pedagogía con mención en Educación y Ciencias Sociales con mención en Desarrollo Local de la extensión en Waslala y que representó un $62 \%$.

\section{Factores que influyen en la deserción estudiantil de URACCAN Recinto Las Minas}

Los principales factores que influyeron en la deserción se agrupan en las dimensiones económicas, familiares, académicas, laborales y vocacionales. En un $60 \%$ adujeron haber abandonado sus estudios de manera voluntaria, relacionando más al factor económico.

El factor económico está estrechamente relacionado con el familiar y laboral, en donde los/as estudiantes desertores expresaron que abandonaron los estudios por que debían desarrollar alguna actividad laboral durante sus estudios, porque no contaban con suficientes recursos económicos para sostener la familia y sus estudios, además de provenir de hogares en condiciones socioeconómicas desfavorables. En algunos casos ellas o ellos sentían que no tenían tiempo para dedicarse al estudio y trabajo, aparte 


\section{LA EDUCACIÓN SUPERIOR EN LA REGIÓN AUTÓNOMA ATLÁNTICO NORTE}

de otros compromisos. Algunos eligieron el trabajo entre ambas opciones, porque les parecía más atractivo en ese momento, aunque con el tiempo consideraron que la decisión no había sido la más acertada.

Algunas y algunos estudiantes al ingresar a estudiar no contaban con un trabajo y dentro del sistema educativo tuvieron la oportunidad de obtenerlo, lo que indica que desempeñarse laboralmente fue más atractivo que seguir en sus estudios. Por otro lado, las responsabilidades se complicaron con los problemas familiares impidiéndoles continuar en sus estudios.

En el factor laboral, el 25\% de las y los entrevistados expresaron que la oportunidad de contar con un trabajo, les obligó a insertarse al mercado laboral.

El factor académico, se dio principalmente cuando aquellas y aquellos ex-estudiantes tuvieron dificultades para aprobar la materia de matemáticas y cálculo. Este aspecto se originó especialmente en el primer y segundo semestre del primer año, en donde se refleja una alta tasa de reprobación.

Características de la universidad: En este aspecto, se mencionan situaciones de la universidad que motivaron al estudiante a retirarse, tales como las condiciones de infraestructura. Aspecto de mayor relevancia en la extensión de Bonanza.

La mayor parte de estudiantes que abandonaron los estudios universitarios lo hicieron en los primeros dos años de la carrera.

Se puede agregar que la pobre orientación hacia la carrera escogida, ha sido otro aspecto que aunque no lo mencionaron, la experiencia nos muestra que cuando ingresan a una carrera a los pocos días, se presentan a la oficina de registro a realizar cambios de carrera, convirtiéndose en un posible candidato a la deserción. Estos estudiantes que no estaban bien orientados en la elección de carrera antes de inscribirse, sin sustentar su decisión en una solida información sobre las mismas y en el proceso de estudio se dieron cuenta de lo que realmente querían, realizaron una mala elección, perdieron el gusto por la carrera y optaron por abandonar los estudios.

Esta distribución pone de manifiesto que son varias las esferas que influyen en la vida estudiantil. Algunas son producto de su historia escolar, otras del medio que les rodea, sea el inmediato, el familiar o el social. En general, puede señalarse que no son factores fáciles de evitar y han tenido tal impacto que los ha orillado a renunciar a concluir su carrera. Es obvio que los jóvenes estudiantes de hoy tienen más compromisos que la sola formación universitaria; que están conscientes de su papel en la 
institución y en la sociedad y, por lo tanto, de la importancia de lograr una combinación ideal entre sus obligaciones escolares, personales y familiares.

\section{Implicancias, sociales e institucionales de la deserción}

La deserción tiene implicancias sociales en términos de las expectativas de los estudiantes y sus familias; implicancias emocionales por la disonancia entre las aspiraciones de sus logros, y también importantes consecuencias económicas tanto para las personas como para el sistema en su conjunto. Adicionalmente, quienes no concluyen sus estudios se encuentran con una situación de empleo desfavorable respecto a quienes terminan. Estas implicaciones son negativas, identificándose las siguientes:

\section{Implicancias sociales:}

Se recalcan aspectos en lo laboral, familiar, académicas y emocionales.

En lo Laboral, se destacan: La obligación de estar titulado para ejercer profesionalmente, baja remuneración económica, rechazo laboral, dificultades para ascender profesionalmente. En lo Familiar: desilusión familiar.

Académicas: dificultades para incorporarse a otra institución por falta de requisitos mínimos, falta de motivación para seguir estudiando, dificultad de adaptación al medio universitario con nuevas exigencias y con condiciones diferentes a la educación secundaria, dificultad de adaptación al medio por parte de estudiantes becados y que pertenecen a grupos indígenas como sumu-mayangna principalmente.

En lo emocional, expresaron haberse sentido frustrados, académica y laboralmente.

\section{Implicancias institucionales}

Para la institución la deserción representó en términos económicos, una inversión fuerte, tomando en cuenta que el o la estudiante que se retiró lo hizo en los tres primeros semestre de la carrera.

Según estimaciones realizadas en relación al costo promedio anual de un estudiante no becado en esta Universidad es de U\$1.957.06 (Un mil novecientos cincuenta y siete dólares con 06/100 centavos.)

Cada una de las personas de la comunidad estudiantil becadas tiene un costo aproximadamente de $\$ 3.000$ (tres mil dólares americanos). No solamente podemos 


\section{LA EDUCACIÓN SUPERIOR EN LA REGIÓN AUTÓNOMA ATLÁNTICO NORTE}

referirnos al costo monetario que implica la deserción, sino también es importante subrayar los costos que no se pueden cuantificar.

El vicerrector Lee expresó al respecto:

“Tomando en cuenta los costos más humanos, implica un profesional menos, un hogar con menos posibilidades de superarse, implica que en un núcleo familiar se le va a ser más difícil salir del círculo de la pobreza, independiente del esfuerzo humano que hizo el profesor. (...) El costo es demasiado alto. ” (Entrevista al Ing. Bismarck Lee León. Vicerrector URACCAN Las Minas).

Se puede inferir que el gasto educativo se pierde, es un gasto sin retorno y finalmente se pierden los recursos humanos calificados, lo cual incide sobre el potencial del desarrollo del país.

La pérdida de estudiantes no sólo implica menores ingresos, sino que también está asociado a un problema de eficiencia y de cumplimento de los planes establecidos así como de la imagen de la universidad en el medio académico.

\section{Propuestas para mantener retención académica}

Sistematicidad en el registro de datos que permitan realizar un análisis posterior de cada periodo académico para el debido seguimiento al problema. Crear un sistema de información que posibilite la toma de decisiones, se proponen las siguientes variables:

- Perfil socio económico y demográfico de los\as aspirantes a las carreras.

- Rendimiento académico de los\as estudiantes que ingresan del nivel medio.

- Asignaturas básicas de las carreras.

- $\mathrm{N}^{\circ}$ de estudiantes promovidos, reprobados, desertores por asignaturas, por año de estudio y por semestre.

- $\quad \mathrm{N}^{0}$ de estudiantes que cambien de carrera.

- $\mathrm{N}^{0}$ de estudiantes inactivos por carrera y semestre y detectarlos una vez ingresen nuevamente al sistema.

- $\quad N^{0}$ de estudiantes que hayan completado el plan de estudio de su carrera en el tiempo requerido.

- Tiempo estimado que lleva un estudiante egresado para graduarse.

44 | CIENCIA E INTERCULtuRaLIDAD, Volumen 4, Año 2, No. 2, Junio 2009 
- Conocer el perfil socioeconómico y demográfico de los aspirantes a las carreras para su debida atención.

- Atención psicológica individualizada a estudiantes becados.

- Mejorar la calidad de la divulgación de las carreras, auxiliándose de materiales como videos de graduados, prácticas de campo, la relación de la universidad con las comunidades donde los estudiantes hacen prácticas de campo.

- Mejoramiento de infraestructura, adquisición de mobiliario, iluminación en aulas, pasillos y entrada al Recinto.

- Realizar un estudio de factibilidad que lleve a las autoridades de la Universidad, visionar nuevas opciones de modalidades en las carreras que se ofertan.

- Atención especial en las asignaturas que presentan más problemas los estudiantes y especial énfasis por etnia, para reducir la reprobación y deserción escolar y lograr índices de aprovechamiento y eficiencia satisfactorios.

- Mejorar los sistemas internos de información sobre los resultados académicos.

- Promover cursos remediales para disminuir la heterogeneidad de los estudiantes que ingresan a la universidad, suplir las deficiencias y dar apoyo adicional a los estudiantes.

- Propiciar el perfeccionamiento pedagógico de los docentes universitarios y el uso de nuevas tecnologías que faciliten el proceso enseñanza-aprendizaje.

\section{Conclusiones}

La deserción se constituye en un problema serio de carácter nacional por cuanto representa una pérdida de recursos humanos, técnicos y de infraestructura, los que consolidados se cuantifican en costos económicos. En su complejidad, la pérdida de recursos humanos hace referencia tanto al personal docente y administrativo que participa en el proceso de formación, como a los estudiantes que salen del sistema y pierden la oportunidad de constituirse en capital humano calificado para el desarrollo nacional.

Los datos presentados dan evidencia del índice de deserción en la URACCAN Recinto Las Minas, los factores que inciden, así como las implicaciones que tiene, tanto para la universidad y el país en general, como para los individuos. 


\section{LA EDUCACIÓN SUPERIOR EN LA REGIÓN AUTÓNOMA ATLÁNTICO NORTE}

Es importante añadir a las recomendaciones ya mencionadas para disminuir la deserción en la educación superior, la necesidad de continuar en la divulgación y orientación vocacional.

\section{Lista de referencias}

Brea, M. (2004). Deserción en la Educación Superior Pública de la República Dominicana. Informe de Avance. Seminario Internacional "Rezago y deserción en la educación superior”. Obtenido el 19 de diciembre del 2007 en http://www.iesalc.unesco. org.ve/programas/Deserción/Deserción\%20-\%20Rep.Dominicana.pdf

CRECALC-UNESCO Caracas (1988). La educación superior en Nicaragua. Obtenido el 10 de enero del 2007 en: httpunesdoc.unesco.orgimages0008000847084763so.pdf

Escobar, Vielka; Delgado Nixa; Fruto, Mayra \& Hernández Dolores. (2005). Estudio Sobre La Deserción y Repitencia En La Educación Superior en Panamá. Presentación. Seminario Internacional "Rezago y deserción en la educación superior". 12 de enero del 2007 en: http://www.iesalc.unesco.org

García, Huidoro (1975). Reflexión sobre la deserción en Costa Rica. San José Costa Rica.

Jaramillo, J. (1999). Los estudios del desempeño estudiantil. Aspectos metodológicos obtenido el 13 de enero del 2007 en: http://www.luz.edu.ve/Portal/repository/ CCP/Doc/Los\%20Estudios\%20del\%20Desempe\%C3\%B1o\%20Estudiantil.pdf

Méndez, B. (2005). Seminario Internacional Rezago y Deserción en la Educación Superior. Resumen del seminario Internacional "Rezago y deserción en la educación superior" Talca, Chile 2005. Obtenido el 12 de enero del 2007 en: http://www.iesalc.unesco.org.ve

Sandino, A. (2006). El Rostro Actual de la Educación Superior en Nicaragua. Obtenido el 13 d enero del 2007: http://www.lpp-uerj.net/olped/exibir opiniao. asp?codnoticias $=19964$

Salgado, R. y Cruz, C. (2004) Deserción en estudiantes de primer ingreso de la URACCAN-Siuna. Nicaragua.

46 | CIENCIA E INTERCULtURALIDAD, Volumen 4, Año 2, No. 2, Junio 2009 